\title{
Oxidative stress and kidney injury in trans-radial catheterization
}

\author{
KONSTANTINOS TSAROUHAS $^{1 *}$, CHRISTINA TSITSIMPIKOU ${ }^{2 *}$, XRISOULA PAPANTONI $^{3 * *}$, \\ DIMITRA LAZARIDOU ${ }^{3 * *}$, MICHAEL KOUTOUZIS $^{4 * *}$, SAVVAS MAZZARIS $^{5^{* *}}$, RAMIN REZAEE $^{6}$, \\ CHARALAMBOS MAMOULAKIS ${ }^{7}$, PANAGIOTIS GEORGOULIAS ${ }^{8}$, CHARITINI NEPKA $^{9}$, \\ ELIAS RENTOUKAS ${ }^{5}$, ZENON KYRIAKIDES ${ }^{4}$, ARISTIDIS TSATSAKIS ${ }^{10}$, \\ DEMETRIOS A. SPANDIDOS ${ }^{11}$ and DEMETRIOS KOURETAS ${ }^{3}$
}

\footnotetext{
${ }^{1}$ Department of Cardiology, University Hospital of Larissa, 41110 Larissa; ${ }^{2}$ General Chemical State Laboratory of Greece, 11521 Athens; ${ }^{3}$ Department of Biochemistry-Biotechnology, School of Health Sciences, University of Thessaly, 41500 Larissa; ${ }^{4}$ 2nd Department of Cardiology, Red Cross Hospital, 11526 Athens; ${ }^{5}$ Department of Cardiology, Amalia Fleming General Hospital, 15127 Athens, Greece; ${ }^{6}$ Clinical Research Unit, Faculty of Medicine, Mashhad University of Medical Sciences, Mashhad, Iran; ${ }^{7}$ Department of Urology, Medical School, University of Crete, 70013 Heraklion; Departments of ${ }^{8}$ Nuclear Medicine and ${ }^{9}$ Pathology, University Hospital of Larissa, 41110 Larissa;

${ }^{10}$ Laboratory of Toxicology, and ${ }^{11}$ Department of Virology, Medical School, University of Crete, 70013 Heraklion, Crete, Greece
}

Received January 26, 2018; Accepted February 28, 2018

DOI: 10.3892/br.2018.1071

\begin{abstract}
Oxidative stress is linked to coronary artery disease and is a major mechanism in contrast-induced nephropathy. Trans-radial approach in coronary angiography (CA) with minimized peri-procedural bleeding is expected to reduce acute kidney injury incidence. In the present study, oxidative stress patterns observed in radial CA and their associations with early manifestations of kidney injury are described. A total of 20 stable coronary disease patients submitted to CA and 17 sex-matched patients undergoing computed tomography for myoskeletal reasons were enrolled. Reduced glutathione, catalase, thiobarbituric acid reactive species (TBARS) levels and total anti-oxidant status were measured at various time points postangiography. In ischemic patients baseline TBARS levels were 2-fold lower compared to controls, while carbonyls levels were $35 \%$ higher. Glutathione was almost 4 -fold lower than the control group. Glutathione and lipid peroxidation in ischemic patients gradually increased after contrast medium administration and reached $180 \%(\mathrm{P}<0.001)$ and $20 \%(\mathrm{P}=0.021)$ after 4-6 h, respectively. Four patients presented early
\end{abstract}

Correspondence to: Dr Konstantinos Tsarouhas, Department of Cardiology, University Hospital of Larissa, Terma Mezourlo, 41110 Larissa, Greece

E-mail: ktsarouhas14@yahoo.gr

${ }^{*, * *}$ Contributed equally

Key words: oxidative stress, contrast-induced nephropathy, transradial catheterization evidence of contrast-induced nephropathy postangiography, while no control patient developed acute kidney injury. In the multiple logistic regression analysis, only the creatinine levels at baseline influenced the frequency of early contrast-induced nephropathy development ( $\beta=0.36,95 \%$ CI: $0.285-0.438$, $\mathrm{P}=0.01$ ). Glutathione low levels were dominant in the baseline values of ischemic patients who developed contrast-induced nephropathy. Glutathione levels rapidly increased while protein oxidation decreased at the expense of lipid peroxidation. In conclusion, early oxidative stress changes occur in trans-radial CA patients with a mild profile, sufficient to mobilize patient antioxidant defenses.

\section{Introduction}

Oxidative stress and coronary artery disease (CAD) are thought to be closely linked (1-3). In the era of trans-radial approach in coronary angiography (CA), where peri-procedural bleeding is minimized, new expectations for reduced incidence of acute kidney injury arise $(4,5)$. It is possible that the site of vascular access and reduced bleeding contributes to reduced incidence of contrast-induced nephropathy (CIN) in radial CA, as was evident in the analysis of the large retrospective Blue Cross Blue Shield of Michigan Cardiovascular Consortium database (6).

However, the importance of additional parameters, such as peri-procedural oxidative stress variations and their associations with early indications of CIN in radial catheterization deserve further exploring. Oxidative stress is linked both to CAD and acute coronary syndromes and at the same time is one of the core features of CIN (7). Oxidative stress variations occurring early post-contrast administration and their 
associations with the contrast volume used may be of interest both from a pathophysiological and clinical perspective. At the same time differences in oxidative stress and occurrence of CIN in patients receiving intravenous contrast media compared to patients with arterial administration of contrast are postulated (8). To some extent dose-toxicity relationship following intravenous administration at usual diagnostic doses seems not to apply (9).

Early recognition of contrast-induced acute kidney injury is of high clinical importance especially with the short hospitalization time currently employed for patients scheduled for CA or intervention. Previously, early renal dysfunction was reported to occur only a few hours post-CA $(10,11)$.

The aim of the present study was to determine the early changes in oxidative status of CAD patients submitted to trans-radial angiography and their association with early signs of CIN compared to the relevant oxidative status of age-sex matched patients submitted to intravenous contrast administration in the setting of computed tomography (CT).

\section{Materials and methods}

Participants. Twenty ischemic patients (CAD group) who were diagnosed with stable CAD and submitted to scheduled CA at the Red Cross General Hospital of Athens were enrolled in the present study. In 4 patients single vessel percutaneous coronary intervention (PCI) was performed. Seventeen sex-matched patients not-diagnosed with ischemia and undergoing CT for myoskeletal reasons at the Amalia Fleming General Hospital (Athens, Greece), were recruited as the control group. The study population had a statistically similar profile concerning underlying pathologies and a similar biochemical and metabolic profile (Table I). Exclusion criteria included untreated peripheral vascular disease, cognitive impairment, acute systematic or infectious diseases or fever, acute pericarditis or myocarditis, new onset atrial fibrillation, on-going maintenance dialysis or impaired kidney function, neoplastic diseases, severe liver dysfunction, major surgery, chronic inflammatory diseases and systemic lupus erythematosus. Patients suffering from severe life-threatening injuries to other organs were also excluded. Written informed consent was obtained from all the participants. The research Ethics Committees of the involved institutes approved the procedures. The Declaration of Helsinki (2000) and the applicable national standards as they relate to the involvement of human subjects in research were enforced. The present study was conducted in the framework of the master theses of DL and XP for the MSc course of Toxicology at the University of Thessaly.

The patients were administered with Ultravist sol $62.34 \%$ (30\% iodine, iopromide) for contrast medium (CM), 50-200 ml intra-arterially for the CAD group and 50-100 ml intravenously for the control group.

CIN is defined as the impairment of renal function manifested by an increase of creatinine of $0.5 \mathrm{mg} / \mathrm{dl}$ or $25 \%$ from baseline within $48-72 \mathrm{~h}$ post-contrast administration $(7,11,12)$. In the present study, early CIN was defined as fulfilling the CIN criteria definition at 6-10 h post-contrast administration. Early renal dysfunction was reported in a previous study as early as $12-18 \mathrm{~h}$ post-contrast administration (11).
Data collection and analyses. Venous blood samples were drawn from the subjects upon hospital admission (baseline values, fasting venous samples), $1 \mathrm{~h}$ after the $\mathrm{CM}$ administration and at the time the patients were discharged (4-6 h for CAD patients and 5-10 $\mathrm{h}$ for the control group), for the evaluation of the general metabolic profile, as well as for the markers of oxidative stress.

In serum samples separated from the total blood, general metabolic parameters were assayed using enzymatic commercial kits and the COBAS INTEGRA 800 automated system by Roche Diagnostics Corp (Indianapolis, IN, USA) using all relevant diagnostic reagents. Serum insulin levels were measured using a human immunoradiometric assay diagnostic kit (KIP1251; DIAsource ImmunoAssays S.A., Belgium). The fasting insulin resistance index (FIRI), which is derived from fasting plasma insulin and glucose levels and has been validated against the hyperinsulinemic-euglycemic clamp (13), was used in the present study as an empirical insulin resistance index. The following formula was used: FIRI = fasting glucose $(\mathrm{mmol} / \mathrm{l}) \mathrm{x}$ fasting insulin $(\mathrm{mU} / \mathrm{l}) / 25(14,15)$.

Plasma samples and suspension of erythrocytes lysates were frozen and kept at $-80^{\circ} \mathrm{C}$ for one month. All the samples (CAD and controls) were analyzed at the same time for the markers of oxidative stress.

Reduced glutathione (GSH), catalase, protein carbonyls, thiobarbituric acid reactive species (TBARS) and total anti-oxidant capacity (TAC) were determined as previously described (16).

Statistical analysis. Results are presented as mean \pm standard deviation (SD). Statistical analyses were performed using SPSS 22.0 software (IBM Corp., Armonk, NY, USA). Significant differences between means for the same parameters were investigated using repeated measures ANOVA with Bonferroni post-hoc test and paired t-test analyses. Independent t-tests were used to compare mean values between groups. Pearson's and Spearman's correlations and linear regression analysis were conducted to investigate associations between various variables. Differences between categorical variables were assessed by the Chi-square test. Multiple linear regression analyses were performed to evaluate the influence of various biochemical parameters at baseline (age, body mass index, FIRI, Pt/L, N/L, haematocrit, haemoglobin, cholesterol, HDL, LDL, triglycerides, creatinine, urea, uric acid, TAC, GSH, TBARS, carbonyls, catalase and chronic medication used), on the elevation of creatinine after $\mathrm{CM}$ administration. $\mathrm{P} \leq 0.05$ was considered to indicate a statistically significant difference.

\section{Results}

Demographics and baseline values. Baseline values for all the parameters screened are summarised in Table I. The oxidative status of CAD patients undergoing scheduled CA was significantly different from the control group. Although the TAC was practically similar between the study groups, it seems that in CAD patients, lipid peroxidation as depicted by TBARS, was 2-fold lower compared to controls, at the expense of protein oxidation, as carbonyls levels were $35 \%$ higher in CAD patients. GSH and catalase were both decreased in CAD patients possibly due to impaired oxygenation. 
Table I. Demographic characteristics and baseline biochemical parameters of the study population.

\begin{tabular}{|c|c|c|c|}
\hline Parameters & CAD patients undergoing CA & $\begin{array}{c}\text { Control group: } \\
\text { No CAD patients undergoing CT }\end{array}$ & P-value \\
\hline No. & 20 & 17 & \\
\hline Age (years) & $64.8 \pm 10.4$ & $71.9 \pm 14.3(\mathrm{P}=0.089)$ & \\
\hline \multicolumn{4}{|l|}{ Sex } \\
\hline Male & 13 & 12 & \\
\hline Female & 7 & 5 & \\
\hline Weight (kg) & $81.5 \pm 10.1$ & $72.4 \pm 9.39$ & 0.009 \\
\hline Height $(\mathrm{cm})$ & $169 \pm 8.15$ & $164 \pm 4.56$ & 0.569 \\
\hline BMI & $28.6 \pm 2.89$ & $25.6 \pm 6.32$ & 0.013 \\
\hline Smoking & 13 (3 ex-smokers) & 9 & 0.569 \\
\hline \multicolumn{4}{|l|}{ Chronic treatment } \\
\hline Anti-platelets & 13 & 10 & \\
\hline Anti-coagulants & 4 & 4 & \\
\hline$\beta$-blockers & 12 & 10 & \\
\hline Ca channel blockers & 7 & 9 & \\
\hline ACE inhibitors & 9 & 6 & \\
\hline Diuretics & 8 & 8 & \\
\hline Statins - antilipidemics & 14 & 13 & \\
\hline Anti-diabetics & 3 & 3 & \\
\hline \multicolumn{4}{|l|}{ Laboratory data } \\
\hline Hematocrit & $38.3 \pm 4.21$ & $39.1 \pm 6.58$ & 0.183 \\
\hline Hemoglobin & $12.7 \pm 1.85$ & $13.1 \pm 1.15$ & 0.325 \\
\hline $\mathrm{P} / \mathrm{L}$ & $92.4 \pm 23.3$ & $93.4 \pm 26.6$ & 0.111 \\
\hline $\mathrm{N} / \mathrm{L}$ & $2.38 \pm 0.714$ & $2.33 \pm 0.324$ & 0.563 \\
\hline Glucose (mg/dl) & $115 \pm 26.9$ & $126 \pm 46.1$ & 0.842 \\
\hline Insulin & $16.3 \pm 11.9$ & $12.4 \pm 8.41$ & 0.278 \\
\hline FIRI & $73.6 \pm 46.9$ & $76.4 \pm 63.0(20.4-180)$ & 0.456 \\
\hline Creatinine $(\mathrm{mg} / \mathrm{dl})$ & $0.796 \pm 0.217$ & $1.06 \pm 0.190$ & 0.001 \\
\hline Urea (mg/dl) & $38.8 \pm 13.4$ & $42.8 \pm 23.2$ & 0.518 \\
\hline Uric acid (mg/dl) & $5.59 \pm 1.67$ & $4.99 \pm 2.43$ & 0.509 \\
\hline Cholesterol (mg/dl) & $188 \pm 49.7$ & $194 \pm 59.7$ & 0.183 \\
\hline Triglycerides (mg/dl) & $132 \pm 45.1$ & $145 \pm 45.1$ & 0.286 \\
\hline $\mathrm{HDL}(\mathrm{mg} / \mathrm{dl})$ & $46.3 \pm 14.8$ & $56.3 \pm 34.8$ & 0.100 \\
\hline LDL (mg/dl) & $106 \pm 32.6$ & $116 \pm 44.6$ & 0.177 \\
\hline $\mathrm{K}(\mathrm{mmol} / \mathrm{l})$ & $4.44 \pm 0.562$ & $4.65 \pm 0.666$ & 0.683 \\
\hline $\mathrm{Na}(\mathrm{mmol} / \mathrm{l})$ & $138 \pm 2.70$ & $135 \pm 6.70$ & 0.983 \\
\hline Albumin (g/dl) & $41.0 \pm 3.92$ & $33.0 \pm 3.92$ & 0.226 \\
\hline Total protein $(\mathrm{g} / \mathrm{dl})$ & $65.6 \pm 5.61$ & $60.4 \pm 1.11$ & 0.345 \\
\hline SGOT (IU/l) & $20.5 \pm 6.59$ & $28.5 \pm 2.49$ & 0555 \\
\hline SGPT (IU/l) & $19.6 \pm 9.44$ & $29.5 \pm 10.4$ & 0.145 \\
\hline$\gamma-\mathrm{GT}(\mathrm{IU} / \mathrm{l})$ & $13.4 \pm 2.23$ & $16.4 \pm 5.23$ & 0.445 \\
\hline LDH (IU/l) & $194 \pm 56.9$ & $204 \pm 69.9$ & 0.256 \\
\hline CPK (U/1) & $121 \pm 154(38-596)$ & $111 \pm 16.3$ & 0.888 \\
\hline \multicolumn{4}{|l|}{ Oxidative stress markers } \\
\hline $\mathrm{GSH}(\mu \mathrm{mol} / \mathrm{g} \mathrm{Hb})$ & $0.516 \pm 0.482$ & $1.91 \pm 0.807$ & $<0.001$ \\
\hline TAC (mmol DPPH/L plasma) & $0.894 \pm 0.128$ & $0.956 \pm 0.150$ & 0.183 \\
\hline TBARS $(\mu \mathrm{mol} / \mathrm{l})$ & $3.66 \pm 1.31$ & $7.32 \pm 1.34$ & $<0.001$ \\
\hline Catalase (U/mg Hb) & $174 \pm 30.1$ & $192 \pm 67.8$ & 0.301 \\
\hline Carbonyls (nmol/mg protein) & $1.22 \pm 0.427$ & $0.793 \pm 0.141$ & $<0.001$ \\
\hline
\end{tabular}

Data presented as mean \pm standard deviation $(\mathrm{SD})$. Statistical comparison is made between groups 1 and 2 . ${ }^{\mathrm{a}} \mathrm{P}<0.05$; ${ }^{\mathrm{b}} \mathrm{P}<0.01$. ACE, angiotensin converting enzyme; BMI, body mass index, BMI=weight $(\mathrm{kg}) /(\text { height })^{2}\left(\mathrm{~m}^{2}\right)$; CAD, coronary artery disease; CT, computed tomography; CA, coronary angiography; ARB, angiotensin receptor blockers; N/L, neutrophils to lymphocytes ratio; P/L, platelets to lymphocytes ratio; CRP, C-reactive protein; FIRI, (glucose $\mathrm{x}$ insulin)/25; TAC, total anti-oxidant activity; GSH, glutathione; TBARS, thio-barbituric acid reactive species. 
A

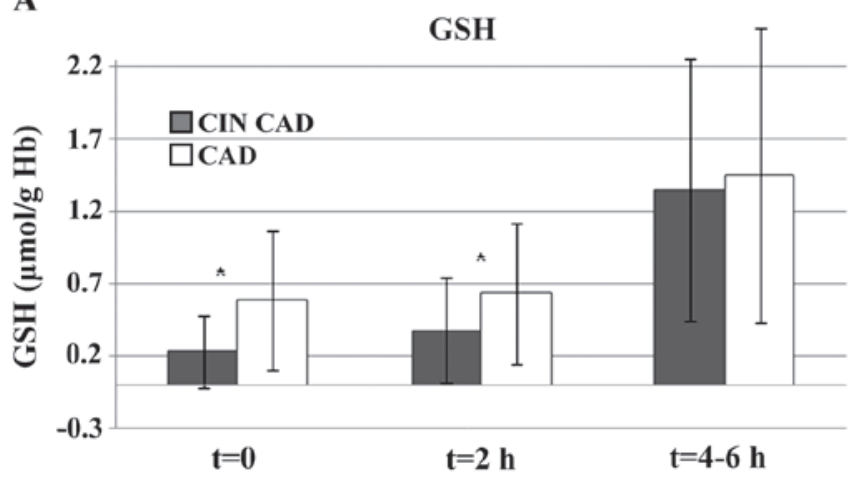

C

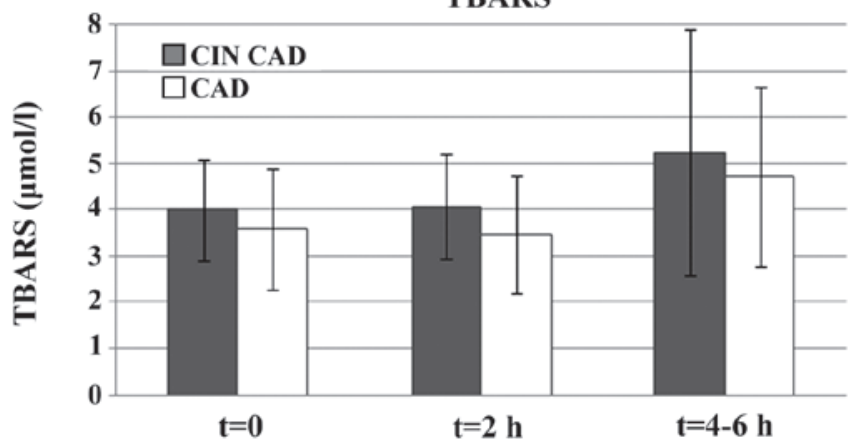

B

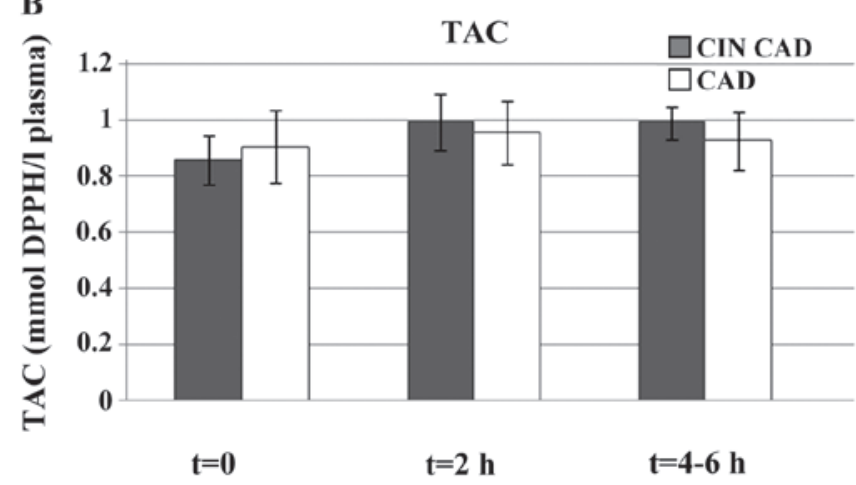

D

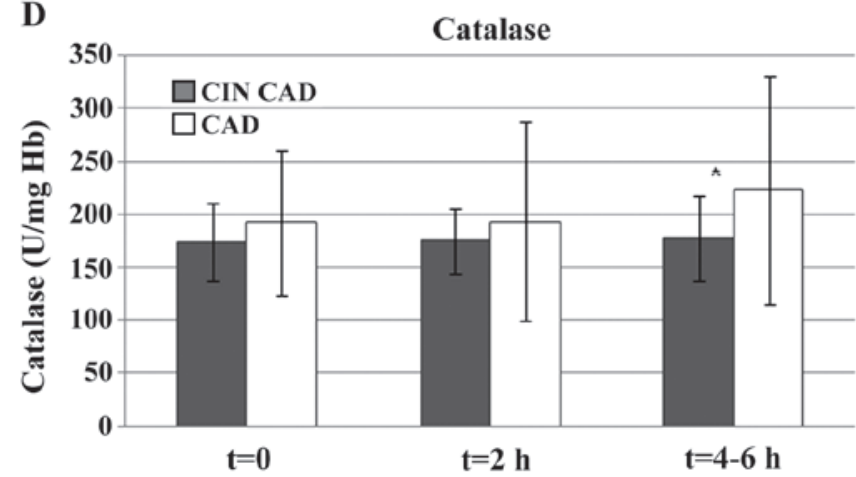

$\mathbf{E}$

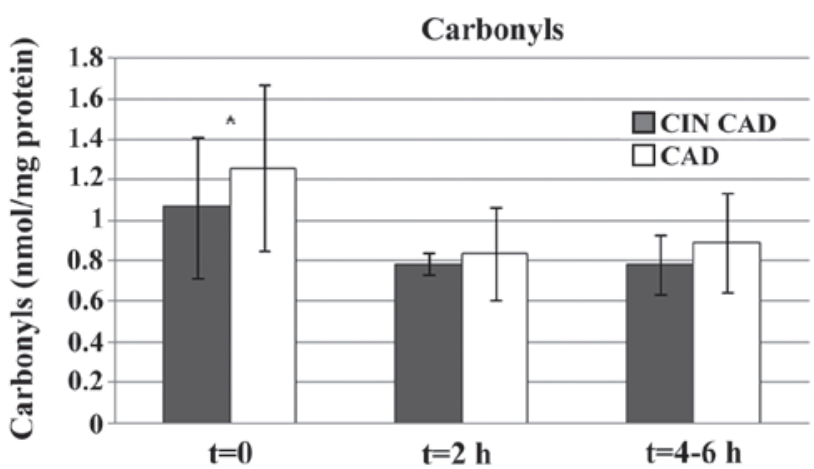

Figure 1. Oxidative stress markers in CAD patients who presented evidence of early CIN and the rest of the CAD group at various time points post-coronary angiography. (A) GSH, (B) TAC, (C) TBARS, (D) Catalase, and (E) carbonyls. GSH, reduced glutathione; TAC, total anti-oxidant capacity; TBARS, thiobarbituric acid reactive species; CAD, coronary artery disease; CIN, contrast-induced nephropathy.

More specifically, in CAD patients, GSH was almost 4-fold lower than the control group, while catalase was only $10 \%$ decreased.

Development of CIN. In 4 of 20 CAD patients CIN was developed early after CM intra-arterial administration during CA. The profile of those patients is presented in Table II. Creatinine increase varied from 36.0 to $77.8 \%$ in $4-6 \mathrm{~h}$ after $\mathrm{CM}$ administration. A constant $16 \%(\mathrm{P}=0.039)$ increase in TAC, at all time intervals, was observed. The most pronounced increase in oxidative stress markers was found for GSH $(41.2-277 \%$ at $1 \mathrm{~h}, \mathrm{P}<0.01$ ), which continued to rise even at $4-6 \mathrm{~h}$ after $\mathrm{CM}$ administration and reached 10 -fold higher values compared to baseline for two individuals. Lipid peroxidation increased with time in 3 of 4 CAD patients that developed early CIN after $\mathrm{CM}$ administration $(1.49-20.5 \%$ at $1 \mathrm{~h}, \mathrm{P}=0.06 ; 12.5-64.5 \%$ at 4-6 h, $\mathrm{P}=0.022$ ). Higher (approximately $6.84 \%$ ) levels were also observed for catalase at $1 \mathrm{~h}$ after $\mathrm{CM}$ administration, which returned to baseline 2-4 h later. Carbonyls, resulting from protein oxidation, decreased up to $56 \%$ in one individual $1 \mathrm{~h}$ after $\mathrm{CM}$ administration, while 2-4 $\mathrm{h}$ later the decrease continued (3.83-64.8\%, $\mathrm{P}=0.045)$.

In multiple logistic regression analysis, only the creatinine levels at baseline influenced the frequency of early CIN development ( $\beta=0.36,95 \%$ CI: 0.285-0.438, $\mathrm{P}=0.01$ ). More specifically, early CIN patients had $11.7 \%$ lower creatinine levels than the rest of the CAD group. In addition, the decreased (61\%) GSH baseline levels exhibited a nearly significant effect $(\mathrm{P}=0.067)$ on the frequency of early CIN development. However the intra-individual variation was very high.

The observed differences in oxidative stress markers between CAD patients who presented evidence of early CIN and the rest of the CAD group at the various time points are shown in Fig 1. 
Table II. Demographic, biochemical and redox profile of CAD patients that have developed early CIN.

\begin{tabular}{|c|c|c|c|c|}
\hline Parameters & Patient 1 & Patient 2 & Patient 3 & Patient 4 \\
\hline Age (years) & 67 & 76 & 80 & 55 \\
\hline Sex & Male & Female & Female & Male \\
\hline Weight (Kg) & 74 & 70 & 73 & 98 \\
\hline Height (cm) & 175 & 165 & 150 & 180 \\
\hline BMI & 24.2 & 25.7 & 32.4 & 30.2 \\
\hline Smoking & Ex-smoker & No & No & Yes \\
\hline \multicolumn{5}{|l|}{ Chronic treatment } \\
\hline Anti-platelets & Yes & Yes & Yes & Yes \\
\hline Anti-coagulants & - & - & - & - \\
\hline$\beta$-blockers & Yes & Yes & - & - \\
\hline Ca channel blockers & - & - & - & Yes \\
\hline ACE inhibitors & Yes & Yes & Yes & - \\
\hline Diuretics & - & Yes & - & - \\
\hline Statins - anti-lipidemics & Yes & Yes & Yes & Yes \\
\hline Anti-diabetics & - & - & - & Yes \\
\hline \multicolumn{5}{|l|}{ Laboratory data } \\
\hline Hematocrit & 37.640 .5 & 38.2 & - & \\
\hline Hemoglobin & 12.313 .3 & 12.5 & - & \\
\hline $\mathrm{P} / \mathrm{L}$ & 11672.4 & 119 & - & \\
\hline $\mathrm{N} / \mathrm{L}$ & 2.94 & 1.39 & 2.79 & - \\
\hline Glucose (mg/dl) & 81 & 106 & 148 & 85 \\
\hline Insulin & 20.2 & 18.5 & 21.6 & 61 \\
\hline FIRI & 65.4 & 78.4 & 128 & 207 \\
\hline \multicolumn{5}{|l|}{ Creatinine (mg/dl) } \\
\hline $\mathrm{t}=0$ & 0.59 & 0.45 & 0.50 & 0.68 \\
\hline $\mathrm{t}=2 \mathrm{~h}$ & 0.89 & 0.68 & 0.65 & 0.98 \\
\hline$t=4-6 h$ & 0.85 & 0.80 & 0.68 & 1.06 \\
\hline \multicolumn{5}{|l|}{ Urea (mg/dl) } \\
\hline $\mathrm{t}=0$ & 31 & 30 & 55 & 16 \\
\hline $\mathrm{t}=2 \mathrm{~h}$ & 42 & 27 & 57 & 25 \\
\hline$t=4-6 h$ & 40 & 30 & 49 & 19 \\
\hline Uric acid (mg/dl) & 5.40 & 4.10 & 3.90 & 4.40 \\
\hline Cholesterol (mg/dl) & 148 & 194 & 182 & 132 \\
\hline Triglycerides (mg/dl) & 132 & 140 & 122 & 152 \\
\hline $\mathrm{HDL}(\mathrm{mg} / \mathrm{dl})$ & 42 & 39 & 56 & 40 \\
\hline $\mathrm{LDL}(\mathrm{mg} / \mathrm{dl})$ & 100 & 116 & 108 & 122 \\
\hline $\mathrm{K}(\mathrm{mmol} / \mathrm{l})$ & 4.9 & 4.0 & 4.4 & - \\
\hline $\mathrm{Na}(\mathrm{mmol} / \mathrm{l})$ & 138 & 136 & 137 & - \\
\hline Albumin (g/dl) & 44 & 36 & - & - \\
\hline Total protein $(\mathrm{g} / \mathrm{dl})$ & 65.2 & 60.4 & - & - \\
\hline SGOT (IU/l) & 19 & 18 & 14 & - \\
\hline SGPT (IU/l) & 20 & 13 & 11 & - \\
\hline$\gamma$-GT (IU/l) & - & 16 & - & - \\
\hline LDH (IU/1) & - & 218 & - & - \\
\hline CPK (U/1) & 59 & 135 & - & - \\
\hline \multicolumn{5}{|l|}{$\begin{array}{l}\text { Oxidative stress markers } \\
\text { GSH }(\mu \mathrm{mol} / \mathrm{g} \mathrm{Hb})\end{array}$} \\
\hline $\mathrm{t}=0$ & 0.663 & 0.145 & 0.061 & 0.045 \\
\hline $\mathrm{t}=2 \mathrm{~h}$ & 1.005 & 0.248 & 0.086 & 0.170 \\
\hline$t=4-6 h$ & 2.626 & 0.270 & 1.23 & 1.48 \\
\hline
\end{tabular}


Table II. Continued.

TAC (mmol DPPH/L plasma)

\begin{tabular}{|c|c|c|c|c|}
\hline $\mathrm{t}=0$ & 0.919 & 0.757 & 0.791 & 0.961 \\
\hline$t=2 h$ & 0.992 & 0.828 & 1.08 & 1.07 \\
\hline$t=4-6 h$ & 1.02 & 0.897 & 1.05 & 1.00 \\
\hline \multicolumn{5}{|c|}{ TBARS $(\mu \mathrm{mol} / \mathrm{l})$} \\
\hline $\mathrm{t}=0$ & 5.86 & 3.77 & 3.82 & 2.75 \\
\hline$t=2 h$ & 5.75 & 2.74 & 4.62 & 3.34 \\
\hline$t=4-6 h$ & 9.85 & 2.96 & 5.64 & 3.04 \\
\hline \multicolumn{5}{|c|}{ Catalase (U/mg Hb) } \\
\hline $\mathrm{t}=0$ & 154 & 125 & 217 & 199 \\
\hline $\mathrm{t}=2 \mathrm{~h}$ & 160 & 135 & 189 & 218 \\
\hline$t=4-6 h$ & 149 & 128 & 220 & 214 \\
\hline \multicolumn{5}{|c|}{ Carbonyls (nmol/mg protein) } \\
\hline $\mathrm{t}=0$ & 0.826 & 1.02 & 0.779 & 1.64 \\
\hline$t=2 h$ & 0.816 & 0.765 & 0.861 & 0.715 \\
\hline$t=4-6 h$ & 0.824 & 0.983 & 0.746 & 0.586 \\
\hline
\end{tabular}

$\mathrm{ACE}$, angiotensin converting enzyme; BMI, body mass index, BMI, weight $(\mathrm{kg}) /(\text { height })^{2}\left(\mathrm{~m}^{2}\right)$; CAD, coronary artery disease; CT, computed tomography; CA, coronary angiography; ARB, angiotensin receptor blockers; N/L, neutrophils to lymphocytes ratio; P/L, platelets to lymphocytes ratio; CRP, C-reactive protein; FIRI, (glucose $\mathrm{x}$ insulin)/25; TAC, total anti-oxidant activity; GSH, glutathione; TBARS, thio-barbituric acid reactive species.

Table III. Effect of CM administration on renal functions parameters and oxidative stress markers in the study population.

A, CAD group (early CIN patients excluded)

\begin{tabular}{|c|c|c|c|c|c|c|c|}
\hline $\begin{array}{l}\text { Sampling } \\
\text { time }\end{array}$ & $\begin{array}{l}\text { Creatinine } \\
(\mathrm{mg} / \mathrm{dl})\end{array}$ & $\begin{array}{c}\text { Urea } \\
(\mathrm{mg} / \mathrm{dl})\end{array}$ & $\begin{array}{c}\mathrm{GSH} \\
(\mu \mathrm{mol} / \mathrm{g} \mathrm{Hb})\end{array}$ & $\begin{array}{c}\text { TAC (mmol } \\
\text { DPPH/L plasma) }\end{array}$ & $\begin{array}{l}\text { TBARS } \\
(\mu \mathrm{mol} / \mathrm{l})\end{array}$ & $\begin{array}{c}\text { Catalase } \\
(\mathrm{U} / \mathrm{mg} \mathrm{Hb})\end{array}$ & $\begin{array}{c}\text { Carbonyls } \\
\text { (nmol/mg protein) }\end{array}$ \\
\hline $\mathrm{t}=0$ & $0.806 \pm 0.117$ & $39.6 \pm 12.4$ & $0.588 \pm 0.482$ & $0.905 \pm 0.128$ & $3.58 \pm 1.31$ & $174 \pm 30.1$ & $1.26 \pm 0.412$ \\
\hline $\mathrm{t}=1 \mathrm{~h}$ & $0.828 \pm 0.192$ & $38.4 \pm 14.8$ & $0.63 \pm 0.493$ & $0.956 \pm 0.114^{\mathrm{b}}$ & $3.46 \pm 1.26$ & $179 \pm 45.1$ & $0.840 \pm 0.228^{b}$ \\
\hline$t=4-6 h$ & $0.876 \pm 0.222$ & $34.1 \pm 11.4^{\mathrm{a}}$ & $1.45 \pm 1.02^{\mathrm{c}}$ & $0.926 \pm 0.105$ & $4.71 \pm 1.94^{\mathrm{a}}$ & $163 \pm 35.1^{\mathrm{a}}$ & $0.889 \pm 0.248^{\mathrm{a}}$ \\
\hline
\end{tabular}

B, Control group

\begin{tabular}{lccccccc}
\hline $\begin{array}{l}\text { Sampling } \\
\text { time }\end{array}$ & $\begin{array}{c}\text { Creatinine } \\
(\mathrm{mg} / \mathrm{dl})\end{array}$ & $\begin{array}{c}\text { Urea } \\
(\mathrm{mg} / \mathrm{dl})\end{array}$ & $\begin{array}{c}\text { GSH } \\
(\mu \mathrm{mol} / \mathrm{g} \mathrm{Hb})\end{array}$ & $\begin{array}{c}\text { TAC (mmol } \\
\text { DPPH/L plasma })\end{array}$ & $\begin{array}{c}\text { TBARS } \\
(\mu \mathrm{mol} / \mathrm{l})\end{array}$ & $\begin{array}{c}\text { Catalase } \\
(\mathrm{U} / \mathrm{mg} \text { Hb) }\end{array}$ & $\begin{array}{c}\text { Carbonyls } \\
(\mathrm{nmol} / \mathrm{mg} \text { protein })\end{array}$ \\
\hline $\mathrm{t}=0$ & $1.06 \pm 0.190$ & $42.8 \pm 23.2$ & $1.91 \pm 0.807$ & $0.956 \pm 0.150$ & $7.32 \pm 1.34$ & $192 \pm 67.8$ & $0.793 \pm 0.141$ \\
$\mathrm{t}=1 \mathrm{~h}$ & $1.04 \pm 0.151$ & $49.6 \pm 24.0$ & $2.01 \pm 0.43$ & $0.945 \pm 0.157$ & $7.81 \pm 1.88$ & $193 \pm 94.1$ & $0.794 \pm 0.128$ \\
$\mathrm{t}=5-10 \mathrm{~h}$ & $0.839 \pm 0.242^{\mathrm{b}}$ & $39.8 \pm 14.9$ & $2.13 \pm 1.14$ & $0.933 \pm 0.161$ & $8.06 \pm 3.26$ & $223 \pm 108$ & $0.785 \pm 0.180$ \\
\hline
\end{tabular}

Statistical significance compared to $\mathrm{t}=0$; ${ }^{\mathrm{a}} \mathrm{P}<0.05$; ${ }^{\mathrm{b}} \mathrm{P}<0.01$; ${ }^{\mathrm{C}} \mathrm{P}<0.001$. CAD, coronary artery disease; CIN, contrast induced nephropathy; $\mathrm{CM}$, contrast medium; GSH, glutathione; TAC, total anti-oxidant activity; TBARS, thio-barbituric acid reactive species.

Effect of CM administration on renal function and oxidative stress markers. In our study, the renal function and the oxidative status of the patients after CM administration, excluding those that have developed early CIN, are described in Table III. CM administration, either intra-arterially or intravenously, did not significantly affect urea, which was actually decreased, probably due to usual instructed hydration of the patients by the attending physicians prior or after CM administration.
Average creatinine, which was almost $20 \%$ lower in the CAD group, slightly increased after 4-6 h. By contrast, in the control group, which consisted of patients undergoing CT for myoskeletal reasons, creatinine was decreased up to $21 \%$ after 5-10 h. The observed differences in creatinine profile could be due to different hydration instructions given to the two groups of patients: CAD patients are usually hydrated as a pre-conditioning process a couple of days prior to $\mathrm{CA}$, whereas patients 
undergoing CT are usually advised to intensively hydrate after the examination.

Regarding oxidative stress markers, in CAD patients TAC increased almost $10 \%$ after the CA. GSH and lipid peroxidation gradually increased after $\mathrm{CM}$ administration and reached $180 \%(\mathrm{P}<0.001)$ and $20 \%(\mathrm{P}=0.021)$ after $4-6 \mathrm{~h}$, respectively. Protein oxidation significantly decreased (approximately $30 \%$, $\mathrm{P}=0.021)$ with time after $\mathrm{CM}$ administration and the same trend was observed for catalase $(6 \%, \mathrm{P}=0.013)$. In the control group, where intravenous CM administration was used, a more subtle effect in the oxidative stress status was observed, with TAC remaining practically unchanged. The same pattern was followed, however, with gradual non-significant increase in GSH (5.23-11.5\%) and increase in TBARS levels (approximately $7 \%$ ).

\section{Discussion}

Reactive oxygen species (ROS) overproduction is thought to be closely linked to cardiovascular disease and its complications. In a previous study in both stable CAD patients and acute coronary syndrome patients, plasma levels of aminothiols cystine and glutathione were associated with the risk of future death independently and additive to inflammation assessed by hs-CRP (1). ROS generation is attributed to vascular enzymatic and non-enzymatic processes. Enzymatic processes include the uncoupling of nitric oxide synthase (NOS), nicotinamide adenine dinucleotide phosphate oxidase, and xanthine oxidase, while main non-enzymatic ROS sources are the mitochondria, where ROS are produced at complex I and III of the respiratory chain (17). Baseline oxidative stress values of the CAD patients presented evidence of a distinct pattern of oxidative stress variations. Lipid peroxidation was significantly reduced and protein oxidation was augmented compared to age-sex matched controls, while anti-oxidant defences of CAD patients represented mainly by glutathione reduction despite the practically unchanged TAC levels. Weinbrenner et al showed that while lipid peroxidation did not differ significantly between CAD patients and controls, increased oxidized LDL and glutathione peroxide levels characterized CAD patients (18). Moreover, similar to our observations, Li et al found that lipid peroxidation was significantly elevataed in CAD patients compared to controls prior to the initiation of statin treatment (19) and returned to similar to control levels post-treatment.

In the setting of CA and PCI, oxidative stress emerges as a dangerous and often underestimated parameter for CIN provocation, while in clinical studies the first attempts of antioxidant supplementation prior or after CA did not present the expected results in CIN amelioration $(7,20)$. Few studies have examined the changing environment regarding oxidative stress following CA and related interventions $(21,22)$, where femoral assess was used. In the era of growing use of radial assess in CA, a changing post-procedural oxidative profile compared to femoral assess was assumed. In the present study, where radial assess was applied exclusively, early variations in oxidative stress markers were found. In the first hour post-CA in CAD patients who did not develop CIN, TAC significantly increased and carbonyls significantly decreased, while in 4-6 h GSH and TBARS significantly increased and catalase significantly decreased. In the study of Kochiadakis et al total peroxides significantly increased 1 day post-stent implantation only in patients receiving bare-metal stents compared to patients receiving sirolimus-eluting stents (21) and the increase in total peroxide correlated with in-stent late lumen loss. In elderly patients with stable angina submitted to CA and PCI via the femoral route, SzewczykGolec et al found no differences in antioxidant enzymes and malondialdehyde levels (22). However, Ciçek et al reported significant increases in TAC immediately after PCI via the femoral route (23).

CIN post-CA is associated with prolonged hospitalization and in-hospital and long-term mortality $(24,25)$. In an attempt to reduce the incidence of CIN with a single injection of reduced glutathione prior to $\mathrm{CA}$, Wang et al (20) identified that 24-h post-procedure TAC was increased, while MDA levels were reduced. In the present study, TBARS levels increased 4-6 $\mathrm{h}$ post-CA. In the setting of primary PCI for myocardial infarction via the femoral route, Börekçi et al found that patients who developed CIN have lower baseline values of total anti-oxidant status and higher oxidative stress index and total oxidant status compared to non CIN patients (26). In the present study, baseline values of TAC, catalase and TBARS were similar between the early CIN and non-CIN groups, while significant differences were found in GSH and carbonyl levels that were both lower in the early CIN group. Using multivariate logistic regression analysis, Börekçi et al (26) found that oxidative stress index and contrast volume used were independent predictors of CIN, while in the present study only baseline creatinine values predicted early CIN.

Ribichini et al showed that a creatinine increase $12 \mathrm{~h}$ post-contrast media administration is the most sensitive and specific risk factor for regular CIN prediction (27). Burchardt et al, however, showed that $12-18 \mathrm{~h}$ post-CA an increase in creatinine level or a decrease in creatinine clearance and a reduction in glomerular filtration rate involved up to $28 \%$ of patients (10). In the present study, a high incidence of early CIN was observed only $6 \mathrm{~h}$ post-CA. Low absolute baseline creatinine values due to excess hydration or subclinical renal dysfunction could account for the rapid $25 \%$ increase in absolute creatinine values accounting for early CIN in the patient subgroup in the present study.

In the present study of exclusive radial assess, distinct patterns of oxidative stress modifications occurred early post-CA with various differences to previous studies of femoral assess to CA. Predominance of GSH reduction in the baseline values of early CIN patients albeit not relevant in regression analysis needs further exploring. The rapid increase of GSH in all the patients submitted to radial CA in an environment of steady TAC values similar to the control group probably indicates a not too unfavorable environment with respect to oxidative stress in radial CA that allows the timely activation of patients' antioxidant defenses. At the same time, lipid peroxidation depicted by TBARS presented a mild increase, suggesting a subtle distortion of lipid cell components as membranes.

In conclusion, early oxidative stress changes occur in bloodstream of patients submitted to radial CA with relative mild profile, sufficient though to mobilize patient antioxidant defenses. 


\section{Acknowledgements}

Not applicable.

\section{Funding}

No funding was received.

\section{Availability of data and materials}

The datasets used and/or analyzed during the present study are available from the corresponding author on reasonable request.

\section{Authors' contributions}

KT, CT, MK, ER, CN, ZK, AT, DAS and DK substantially contributed to the conception and design of the study. XP, DL, MK, SM, GG, CN, DK participated in the acquisition of reported data. $\mathrm{RR}, \mathrm{CM}, \mathrm{GG}, \mathrm{CN}$ participated in the processing and analysis of reported data. All the authors participated in the interpretation of reported data. All the authors contributed to the writing of the manuscript and participated in the review and interpretation of the data. All the authors read and approved the final manuscript before submission.

\section{Ethics approval and consent to participate}

Written informed consent was obtained from the participants. The research Ethics Committees of the involved institutes approved the procedures. The Declaration of Helsinki (2000) and the applicable national standards as they relate to the involvement of human subjects in research were enforced. The present study was conducted in the framework of the master theses of the students Lazaridou and Papantoni at the MSc course of Toxicology at the University of Thessaly.

\section{Consent for publication}

Not applicable.

\section{Competing interests}

Demetrios Spandidos is the Editor-in-Chief for the journal, but had no personal involvement in the reviewing process, or any influence in terms of adjudicating on the final decision, for this article.

\section{References}

1. Patel RS, Ghasemzadeh N, Eapen DJ, Sher S, Arshad S, Ko YA, Veledar E, Samady H, Zafari AM, Sperling L, et al: Novel biomarker of oxidative stress is associated with risk of death in patients with coronary artery disease. Circulation 133: 361-369, 2016.

2. Vasilaki F, Tsitsimpikou C, Tsarouhas K, Germanakis I, Tzardi M, Kavvalakis M, Ozcagli E, Kouretas D and Tsatsakis AM: Cardiotoxicity in rabbits after long-term nandrolone decanoate administration. Toxicol Lett 241: 143-151, 2016.

3. Ungurianu A, Margină D, Grădinaru D, Băcanu C, Ilie M, Tsitsimpikou C, Tsarouhas K, Spandidos DA and Tsatsakis AM: Lipoprotein redox status evaluation as a marker of cardiovascular disease risk in patients with inflammatory disease. Mol Med Rep 15: 256-262, 2017.
4. Andò G, Cortese B, Frigoli E, Gagnor A, Garducci S, Briguori C, Rubartelli $\mathrm{P}$, Calabrò $\mathrm{P}$ and Valgimigli M; MATRIX investigators: Acute kidney injury after percutaneous coronary intervention: Rationale of the AKI-MATRIX (acute kidney injury-minimizing adverse hemorrhagic events by TRansradial access site and systemic implementation of angioX) sub-study. Catheter Cardiovasc Interv 86: 950-957, 2015.

5. Andò G, Cortese B, Russo F, Rothenbühler M, Frigoli E, Gargiulo G, Briguori C, Vranckx P, Leonardi S, Guiducci V, et al; MATRIX Investigators: Acute kidney injury after radial or femoral access for invasive acute coronary syndrome management: AKI-MATRIX. J Am Coll Cardiol 69: 2592-2603 2017.

6. Kooiman J, Seth M, Dixon S, Wohns D, LaLonde T, Rao SV and Gurm HS: Risk of acute kidney injury after percutaneous coronary interventions using radial versus femoral vascular access: Insights from the blue cross blue shield of Michigan Cardiovascular Consortium. Circ Cardiovasc Interv 7: 190-198, 2014.

7. Mamoulakis C, Tsarouhas K, Fragkiadoulaki I, Heretis I, Wilks MF, Spandidos DA, Tsitsimpikou C and Tsatsakis A: Contrast-induced nephropathy: Basic concepts, pathophysiological implications and prevention strate gies. Pharmacol Ther 180: 99-112, 2017.

8. Katzberg RW and Newhouse JH: Intravenous contrast medium-induced nephrotoxicity: Is the medical risk really as great as we have come to believe? Radiology 256: 21-28, 2010.

9. American College of Radiology: ACR comittee on drugs and contrast media. ACR Manual on Contrast Media: 129, 2016.

10. Burchardt P, Synowiec T, Jerzykowska S, Angerer D and Żurawski J: Early renal dysfunction after iodine contrast administration: New insight into the actual problem. Kardiol Pol 73: 233-234, 2015.

11. Burchardt P, Guzik P, Tabaczewski P, Synowiec T, Bogdan M, Faner P, Chmielarz-Sobocińska A and Palasz A: Early renal dysfunction after contrast media administration despite prophylactic hydration. Int J Cardiovasc Imaging 29: 959-966, 2013.

12. Schweiger MJ, Chambers CE, Davidson CJ, Zhang S, Blankenship J, BhallaNP, Block PC, Dervan JP, Gasperetti C, Gerber L et al: Prevention of contrast induced nephropathy: recommendations for the high risk patient undergoing cardiovascular procedures. Catheter Cardiovasc Interv 69: 135-140, 2007. https://doi.org/10.1002/ccd.20964.

13. AlZadjali MA, Godfrey V, Khan F, Choy A, Doney AS, Wong AK, Petrie JR, Struthers AD and Lang CC: Insulin resistance is highly prevalent and is associated with reduced exercise tolerance in nondiabetic patients with heart failure. J Am Coll Cardiol 53: 747-753, 2009.

14. Duncan MH, Singh BM, Wise PH, Carter G and Alaghband-Zadeh J: A simple measure of insulin resistance. Lancet 346: 120-121, 1995.

15. Tsarouhas K, Tsitsimpikou C, Haliassos A, Georgoulias P, Koutsioras I, Kouretas D, Kogias J, Liosis I, Rentoukas E and Kyriakides Z: Study of insulin resistance, TNF- $\alpha$, total antioxidant capacity and lipid profile in patients with chronic heart failure under exercise. In Vivo 25: 1031-1037, 2011.

16. Tsamouri MM, Rapti M, Kouka P, Nepka C, Tsarouhas K, Soumelidis A, Koukoulis G, Tsatsakis A, Kouretas D and Tsitsimbikou C: Histopathological evaluation and redox assessment in blood and kidney tissues in a rabbit contrast-induced nephrotoxicity model. Food Chem Toxicol 108: 186-193, 2017. https://doi.org/10.1016/j.fct.2017.07.058.

17. Juni RP, Duckers HJ, Vanhoutte PM, Virmani R and Moens AL: Oxidative stress and pathological changes after coronary artery interventions. J Am Coll Cardiol 61: 1471-1481, 2013.

18. Weinbrenner T, Cladellas M, Isabel Covas M, Fitó M, Tomás M, Sentí M, Bruguera J and Marrugat J: High oxidative stress in patients with stable coronary heart disease. Atherosclerosis 168: 99-106, 2003

19. Li J, Sun YM, Wang LF, Li ZQ, Pan W and Cao HY: Comparison of effects of simvastatin versus atorvastatin on oxidative stress in patients with coronary heart disease. Clin Cardiol 33: 222-227, 2010.

20. Wang C, Wang W, Ma S, Lu J, Shi H and Ding F: Reduced glutathione for prevention of renal outcomes in patients undergoing selective coronary angiography or intervention. J Interv Cardiol 28: 249-256, 2015.

21. Kochiadakis GE, Arfanakis DA, Marketou ME, Skalidis EI, Igoumenidis NE, Nikitovic D, Giaouzaki A, Chlouverakis G and Vardas PE: Oxidative stress changes after stent implantation: A randomized comparative study of sirolimus-eluting and bare metal stents. Int J Cardiol 142: 33-37, 2010 
22. Szewczyk-Golec K, Grzelakowski P, Lugowski T and Kedziora J: The effects of percutaneous transluminal coronary intervention on biomarkers of oxidative stress in the erythrocytes of elderly male patients. Redox Rep 22: 315-322, 2017.

23. Ciçek D, Tamer L, Pekdemir H, Cin VG, Yildirim H, Kara AA and Yurtdaş M: Coronary angioplasty induced oxidative stress and its relation with metoprolol use and plasma homocysteine levels. Anadolu Kardiyol Derg 6: 308-313, 2006.

24. James MT, Ghali WA, Knudtson ML, Ravani P, Tonelli M, Faris P, Pannu N, Manns BJ, Klarenbach SW and Hemmelgarn BR; Alberta Provincial Project for Outcome Assessment in Coronary Heart Disease (APPROACH) Investigators: Associations between acute kidney injury and cardiovascular and renal outcomes after coronary angiography. Circulation 123: 409-416, 2011.

25. McCullough PA: Contrast-induced nephropathy: Definitions, epidemiology, and implications. Interv Cardiol Clin 3: 357-362, 2014.
26. Börekci A, Gür M, Türkoğlu C, Caylı M, Selek S, Kaypaklı O, Uçar H, Coşkun M, Şeker T, Koç M, et al: Oxidative stress and paraoxonase 1 activity predict contrast-induced nephropathy in patients with ST-segment elevation myocardial infarction undergoing primary percutaneous coronary intervention. Angiology 66: 339-345, 2015.

27. Ribichini F, Graziani M, Gambaro G, Pasoli P, Pighi M, Pesarini G, Abaterusso C, Yabarek T, Brunelleschi S, Rizzotti P, et al: Early creatinine shifts predict contrast-induced nephropathy and persistent renal damage after angiography. Am J Med 123: 755-763, 2010

(i)(3) This work is licensed under a Creative Commons Attribution-NonCommercial-NoDerivatives 4.0 International (CC BY-NC-ND 4.0) License. 